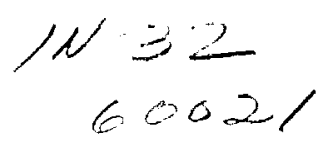

NASA Technical Memorandum 107218 AIAA-96-0993

\title{
Communications Satellites in the National and Global Health Care Information Infrastructure: Their Role, Impact, and Issues
}

John E. Zuzek and Kul B. Bhasin

Lewis Research Center

Cleveland, Ohio

Prepared for the

16th International Communications Satellite Systems Conference cosponsored by AIAA, CASI, AAAF, DGLR, and IEICE

Washington, DC, February 25-29, 1996

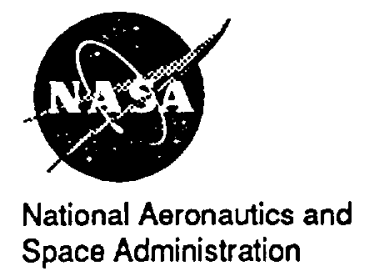


Trade names or manufacturers' names are used in this report for identification only. This usage does not constitute an official endorsement, either expressed or implied, by the National Aeronautics and Space Administration. 


\title{
COMMUNICATIONS SATELLITES IN THE NATIONAL AND GLOBAL HEALTH CARE INFORMATION INFRASTRUCTURE: THEIR ROLE, IMPACT, AND ISSUES
}

\author{
John E. Zuzek" and Kul B. Bhasin \\ NASA Lewis Research Center \\ Cleveland, OH 44135 USA
}

\begin{abstract}
1. Abstract
Health care services delivered from a distance, known collectively as telemedicine, are being increasingly demonstrated on various transmission media. Telemedicine activities have included diagnosis by a doctor at a remote location, emergency and disaster medical assistance, medical education, and medical informatics. The ability of communications satellites to offer communication channels and bandwidth on demand, connectivity to mobile, remote and under served regions, and global access will afford them a critical role for telemedicine applications within the National and Global Information Infrastructure (NIV/GII). The importance that communications satellites will have in telemedicine applications within the NIVGII, the differences in requirements for NII vs. GII, the major issues such as interoperability, confidentiality, quality, availability, and costs, and preliminary conclusions for future usability based on the review of several recent trials at national and global levels are presented.
\end{abstract}

\section{Introduction}

At a recent "National Forum on Military Telemedicine," Brigadier General Russ Zajtchuk gave an opening address in which he stated, "The time is right to change the way we practice medicine." He also stated that we must become aware of and understand all of the dual applications of telemedicine in both practice and technologies. There is currently an explosion in technology that is not being fully utilized, especially in telecommunications. This includes ISDN types of services, terrestrial fiber, and satellite communications.

Telemedicine is delivering health care to patients anywhere in the world through the combined use of communications technology and critical medical expertise. The national health care industry alone is already reaching more than one trillion dollars in business. To make health care reform a reality, the application of technologies such as those in the field of telecommunications can play an important role. Application of telemedicine technologies and practice has the potential to reduce costs and improve care. Telemedicine can offer physicians new opportunities and capabilities to enhance their own skills. Telemedicine can revolutionize the way we deliver health care in this country and around the world.

Routine health care and information services will be offered by both wired and wireless local, medium, and wide area networks (i.e., LANs, MANs, and WANs). However, satellites will provide services to remote national locations, most global locations, mobile units, and airplanes. This scenario is illustrated in the Figure 1. Currently, the applications in medical consultations, disaster care, and medical specialities, such as radiology and pathology are being demonstrated at low data rates. Many of these applications demand multimedia transmission at high data rates to reach their full potential.

The major issues are interoperability, confidentiality, quality of transmission and costs. Interoperability issues are at two levels: the various medical equipment, smart cards, digital assistants, and computers and workstations have to be interoperable with satellite earth stations and mobile antennas. The satellite networks need to achieve seamless interoperability with the fiber and wireless networks. Figure 2 illustrates the layered approach to viewing telemedicine. Satellite communications and other telecommunications media exist at the "telecom" layer and interoperability issues are addressed with the "protocol" and "application" layers. We will examine various items at the "clinical" and "application" layers to find the key telecommunications and interoperability issues in 
telemedicine.

This paper will outline the critical role satellites will play in the National and Global Information Infrastructure for health care applications and will outline the differences in their requirements for NII vs. GII. For example, one difference noted is that medical consultation in the national health care information infrastructure may be hindered by the potential of increased malpractice lawsuits based on transmitted data. Global markets welcome the access to technology and believe this provides timely and quality medicine. Preliminary conclusions drawn from several recent trials at national and global levels are reviewed to project future usability. The medical user's needs are defined for these applications to become prevalent. The technical developments required by these emerging applications, interoperability issues and policy requirements are presented.

\section{Information Infrastructure for Health Care Applications}

The National and Global Information Infrastructure (NIVGII) is envisioned as a seamless web of communication networks composed of different transmission media such as satellites, fiber optic cable, copper wire, coaxial cable and radio that will carry a broad range of telecommunications and information services and information technology applications into homes, businesses, schools, and hospitals'. The national and global interconnectivity is expected to improve the health care access and quality and has the potential to reduce medical costs. As an example, the NIUGII can facilitate health care delivery through telemedicine, linking rural physicians to major medical facilities for off-site consultations on difficult diagnoses. It can provide a data base search and on-line questioning of a consulting medical expert. It can also quicken response time for disaster relief. However, applications of telecommunication networks to medicine beyond the telephone have been under development for many years. In 1959, Wittson et al.. were the first to use a microwave link for telepsychiatry by two-way TV transmission 112 miles away. In 1980, NASA demonstrated telemedicine in the Space Technology Applied to Rural Papago Advanced Health Care (STARPAHC) project. Evaluations of these early programs showed that equipment was effective at transmitting the information needed for most uses and users were satisfied, however, the most important cause of failure was the inability to justify these programs on a cost-benefit basis. Now in the current scenario of telemedicine with the use of advance communication and information technologies the use of telemedicine has expanded. A typical connection between two telemedicine workstations is illustrated in Figure 3.

The telemedicine workstation uses T-1 networks as a minimum and can interconnect to national and global networks via satellite and terrestrial telecommunication links. The workstation has multimedia capabilities meeting the standards of medical community. Digitally compressed video is used to transmit, store and access medical information. The workstation can also a) transfer of a patient's medical records from a common database to requesting doctor $b$ ) patient monitoring according to requesting doctor c) patient due for another checkup d) patient due for a lifesaving prescription drug refill. Such a system can enhance quality of service at reduced cost and providing specialized medical services to remote locations.

Health care applications of an information infrastructure are also needed in variety of mobile situations such as emergency vehicle, aircraft and ship. Communication Satellites are the only means to provide access to the health information infrastructure to mobile users. Additional benefits of availability of telemedicine in mobile situations will be improved emergency and disaster care.

In the emerging health information infrastructure, distance medical education can be provided by interactively linking medical educators, practitioners, students and patients on a global basis. Apart from providing information to the patient on particular disease the major requirement for medical education in the HII is capability to transmit highresolution images. Each image may need up to tens of megabytes of bandwidth. It becomes clear that health care applications have varied requirements and the implementation of each may be cost prohibitive. However, with the development health information infrastructure many of these applications can be addressed.

\section{Technical Requirements and Market Drivers}

Technical requirements of telemedicine applications from the user point of view depend on the service under consideration. As an example, teleradiology, telepathology, medical examination, on-line medical informatics, and realtime medical consultations all have different requirements from the telecommunications standpoint. In Table 1, detailed information on workstation and data rate requirements are presented. High quality video transmission requirements show up in most of them. The data rates requirements range from $\mathrm{T}-1$ to $\mathrm{OC}-3$ rates.

The communication satellites that provide mobile telemedicine services will require high channel capacity and high transmission reliability in real time operation. 


\begin{tabular}{|c|c|c|}
\hline Application/Service & Requirements & Impact \\
\hline Medical Examinations & $\begin{array}{c}\text { Two-Way Video \& } \\
\text { Electronic Stethoscope, Otoscope, } \\
\text { Ophthalmoscope }\end{array}$ & $\begin{array}{c}10 \mathrm{kbps} \text { up to Tl }(1.544 \mathrm{Mbps}) \text { or } \\
\text { higher rates }\end{array}$ \\
\hline Radiology & X-Rays, CT scans, MRI, mammogram, etc. & $\begin{array}{l}\text { File Sizes Range From 1-20 Mbytes. } \\
\text { T1-T3 rates needed }\end{array}$ \\
\hline Pathology & $\begin{array}{l}\text { High Resolution, Color Pathological } \\
\text { Imagery }\end{array}$ & $\begin{array}{l}\text { Large Files (10's of Mbytes) and } \\
\text { High Data Rates }\end{array}$ \\
\hline Medical Education & $\begin{array}{l}\text { One-Way or Two-Way Interactive Audio \& } \\
\text { Video }\end{array}$ & $\begin{array}{l}\text { Point-Multipoint High Quality } \\
\text { Videoconferencing }\end{array}$ \\
\hline Virtual Reality & $\begin{array}{c}\text { Detailed, Digitized 3-D Active Models of } \\
\text { Human Body For Surgical and Other } \\
\text { Training }\end{array}$ & $\begin{array}{l}\text { Large Data Sets Need To Be } \\
\text { Manipulated in Real-Time, } \\
\text { Possibility of OC3 Rates }\end{array}$ \\
\hline Smart Card Medical Data & $\begin{array}{c}\text { Basic Data Transmission to Multimedia } \\
\text { Data Transmission }\end{array}$ & $16 \mathrm{kbps}-1.5 \mathrm{Mbps}$ and higher \\
\hline Computer Aided Diagnosis & TBD & TBD \\
\hline
\end{tabular}

Table 1 - Data requirements for telemedicine equipment operation

The transmitting and receiving system in the moving vehicle must be small and light. The medical work station and associated digital circuitry must be made small.

Ever more of us are spending longer periods of time in a variety of moving vehicles, especially in international travel by aircraft and ships. The number of medical emergencies on aircraft has been on the increase. On the ground, death rates within the first hour, the so called "golden hour" for emergency vehicle users, is very high. The access to the health information infrastructure in the mobile environment will have considerable benefit if it can be provided cost effectively.

It is clear that the cost of equipment associated with telemedicine and, to some extent, the cost of the communication link is coming down. This lower implementation cost makes several applications feasible. In the international market where the cost of travel is high and immediate intervention by the specialist is desired, the access to global information infrastructure by the major hospitals will be mutually beneficial.

A recent survey conducted by the Health care Information and Management Society and Hewlett-Packard Company of information systems professionals at leading Health care organizations identified following applications holding the most promise for Health care organizations:

Education/training - $42 \%$

remote diagnostics - $32 \%$

Telemedicine has a high endorsement $29 \%$ of the Health care organizations had already invested and $30 \%$ had a high priority in setting up telemedicine capabilities. The survey does not separate out the communication media. However, increased use of telemedicine in health care applications will require use of satellite networks where other networks cannot be deployed. The use of satellite networks is foreseen in remote, mobile and global applications. In countries where a free market is taking hold and/or percentage of upper-middle class is rising, access to high quality medical care becomes a priority.

\section{Uses of Satellites in Telemedicine}

Many examples of the use of communications satellites in telemedicine activities can be found both in the Unites States and throughout the world. However, the vast majority of these efforts have been demonstrations or at least experimental in nature. The possibilities of the uses of satellites in telemedicine are quite broad and have yet to be fully explored. 


\subsection{NASA Examoles of Satellite Telemedicine}

One of the earliest demonstrations of telemedicine was using the experimental NASA ATS-1 satellite helping to provide health care to remotely located native Alaskans ${ }^{11}$. This program was begun with the support of the Indian Health Service who was trying to solve the difficult problem of providing health care to widely scattered remote villages. The use of satellite communications was introduced to combat the lack of reliable communications in the Alaskan bush. Initially, the experiment was set up to provide simple teleconsultations to these small, remotely located villages. The system was also used for communications between patients at the actual hospital and their families in the remote villages, which had previously been impossible due to the lack of any telephone service. In 1974-75, another NASA experimental satellite, ATS-6, added a video link to the teleconsultation service that allowed for more possible telemedicine applications such as interactive searching of on-line medical libraries and for the transmission of medical continuing education courses. The experiments using the NASA satellites were so successful that the state of Alaska and the Public Health Service subsequently evolved the experimental system into an operational system using commercial satellite services.

NASA has also been involved in international telemedicine. In December of 1988, a devastating earthquake occurred in the Armenian Republic of the then Soviet Union. The earthquake caused significant loss of life and numerous injuries to the population with devastating destruction and damage to the regional infrastructure. A multinational effort to provide initial medical and material assistance was undertaken immediately. Still, the ability of the local and relief organization physicians to care for the injured was overburdened by the sheer number of casualties and the damage to the communications infrastructure in the area. In early 1989, through a preexisting protocol on the Exploration and Use of Outer Space for Peaceful Purposes, NASA developed and proposed a plan for telemedicine via satellites to the Soviet government. The idea was to provide expert medical consultation from American physicians to their counterparts in Armenia concerning plastic/ reconstructive surgery, physical and psychological rehabilitation, public health, and epidemiology.

The Telemedicine Spacebridge to Armenia was established between several sites in the United States and the Armenian Republic in early 1989 to provide this assistance. Transponder time on an out of service Intelsat satellite was provided for the link from Armenia to the U.S. while a Soviet satellite was used for the link from the U.S. sites to Armenia.
The most recent international telemedicine demonstration was known as the Spacebridge to Moscow and took place in 1993-94. Through the U.S.-U.S.S.R. Joint Working Group on Space Biology and Medicine, an experimental program was developed to explore the viability of deploying small satellite earth stations for disaster relief efforts and to show its utility in providing telemedicine support over great distances to remote areas.

The Telemedicine Spacebridge Demonstration Project provided a two-way audio and full bandwidth video link between medical specialists at participating medical institutions in the U.S. and their counterparts in the Russian Republic. The operational Spacebridge link included such applications as disaster medicine, routine patient consultation, medical evaluation, medical information exchange, space medicine issues, and other telemedicine applications such as telepathology and teleradiology.

Two earth stations, one a domestic Ku-band earth station and the other a Russian earth station for use with the Russian WSDRN (Western Space Data Relay Network) satellite located at $16^{\circ}$ West longitude, were installed at NASA's Lewis Research Center in Cleveland, Ohio. These two stations, coupled with video standard converters and encryption equipment, formed a "gateway facility" to link Moscow, Russia to various participating U.S. medical institutions via a double satellite "hop." A total of 16 sessions took place during an eight month period.

NASA's Advanced Communications Technology Satellite (ACTS) program demonstrates the use of advanced satellite communications technologies through a variety of experiments. The ACTS Experiments Program includes several experiments that test the delivery of health care services to remote locations and demonstrate the use of advanced mobile communications. ACTS is being used to transmit images and information to physicians and specialists for use in remote diagnoses and medical consultation High-resolution medical imagery from X-rays, MRIs, or CT scans can be sent to another location for review by a consulting physician. The ACTS Mobile Terminal (AMT) has also been used to transmit patient data from emergency vehicles while en route to a hospital.

A variety of ACTS experiments are underway that involve at least six medical organizations. One such experiment is the Mobile Radiology Image Transfer experiment by the University of Washington with the cooperation of $\mathrm{GE}$ Medical Systems. The Mobile Radiology Image Transfer experiment links a mobile Computed Tomography (CT) or Magnetic Resonance Imaging (MRI) van to the Department of Radiology at the University of Washington Medical 
Center. While stationary, the mobile van transmits digital medical images from various locations in the state of Washington. This investigation will allow for quality control and for diagnostic consultation and interpretation of the remotely obtained images at a major university medical center. This use of ACTS shows the practical medical application of teleradiology from remote locations to medical centers.

\subsection{Department of Defense Examples}

The United States Department of Defense has a variety of telemedicine projects currently underway ranging from digital patient records to battlefield access to patient information and remote medical care. Many of these projects involve the use of satellite communications assets. For example, the Akamai Project' uses a global network including both the NASA ACTS satellite and another Pacific-based communications satellite to link Tripler Army Hospital in Hawaii with various Pacific Rim sites and to George Washington University to perform a variety of telemedicine activities. The ACTS satellite was also used to support recent telemedicine activities in Haiti and a commercial satellite was used to link Walter Reed Army Medical Center with Zagreb, Croatia.

\subsection{Telemedicine Around the World}

There are many other examples of the use of satellites in telemedicine around the world ${ }^{6}$. Australia had a satellitebased telemedicine project known as the North-West Telemedicine Project that involved five communities who were largely Aboriginal. The major benefit of this project was that fewer patients from Queensland had to be evacuated by the Royal Flying Doctor Service.

Memorial University of Newfoundland, Canada, established a teleconferencing system linking nine communities across the provinces in conjunction with the Canadian Space Program in 1979. Currently there are 220 sites in 120 communities linked together using a communications network that uses standard telephone equipment to provide simultaneous programming.

The French established the Telemedicine Institute in Toulouse, France. Several examples of the use of telemedicine in France can be cited, although most do not involve the use of satellites. In 1984, France established the Communicable Diseases Computer Network (FCDN) to track trends in seven communicable diseases and their treatments. Several European and national health agencies use the FCDN to exchange information. The University of Dijon operates a telepathology system using the French
ISDN network for exchanging images for diagnostic purposes. In addition, the French have been working in the development of medical smart cards and their applications.

Italy has been using telemedicine in various forms since 1970. A formal telemedicine project in emergency medicine was begun in 1982 in a cooperative effort between the national telephone company and the Ministries of Scientific Research and Health. This project and many others within Italy have not yet explored possible satellite applications in telemedicine, although Italy is one of the European leaders in satellite communications itself.

Japan is another world leader in both satellite communications and telemedicine. Kyoto University developed the world's first HDTV-based telemedicine system that uses ISDN to receive high resolution images of frozen pathology sections that can be remotely manipulated. Several teleradiology trials also are occurring throughout Japan. At least one of the Japanese telemedicine efforts is using satellite communications. In 1992, the Telemedicine Society of Japan, which is a non-governmental organization, was founded at Tokai University School of Medicine. This organization is sponsoring the Pan-Pacific Regional Telecommunications Network Experiments and Research Satellite project (PARTNERS). With the cooperation of the PTT and the Japanese space agency (NASDA), PARTNERS uses the ETS-V satellite to link medical institutions in Cambodia, Thailand, Indonesia, Papua New Guinea, Fiji, and the University of Hawaii for telemedicine and educational research.

In Greece, a telemedicine network has been established within the framework of a NATO-Sponsored project. The first phase of this project began in 1988 and created a telemedicine network among its islands using standard telephone lines for transmission of electrocardiograms and $\mathrm{X}$-rays. Since the quality of these links was quite poor, the second phase will use satellite links between three Greek islands and University of Athens using $384 \mathrm{kbps}$ links. Coverage of much of the European continent is planned for later phases.

SatelLife/HealthNet is an international nonprofit organization based in the United States which uses microsatellite technology to provide health communications services to developing nations. SatelLife now provides island communities with some communications capabilities via the low earth orbiting HealthSat 1 and 2 satellites. HealthNet stations are currently operating in 13 countries around the world assisting local practitioners in remote medical care. 


\section{Critical Issues}

The evolving health information infrastructure requires communication satellite networks to be a part of it. Several key issues need to be resolved, as discussed below:

\section{Availability}

Several telemedicine applications require bandwidth on demand. Satellite networks are not readily available to provide bandwidth on demand services. In large disaster emergencies, satellite networks have been temporarily linked together at significant cost, however, general availability for such specialized services is poor.

\section{Cost}

The costs associated with telemedicine are currently high. These costs are associated with access to satellite networks, specialized workstations, and technical personnel to use and maintain these systems on both the transmission and receiver ends. Current perceptions that health care in general is already too expensive may initially slow the move for health care facilities to add telemedicine services. However, satellite communication links have a significant potential to reduce health care costs. Underutilized hospital or speciality services could produce higher revenues by marketing their services to rural and remote areas who are typically without specialty medical access. This could also include patients for whom travel is difficult, too far, or too expensive, as with international patients.

As pointed out by Bashshur ${ }^{34}$, the relationship between telemedicine cost, quality and access and their impact on patient, provider and society are complex. However, without doubt, greater availability with reduced costs of access and workstations will be pivotal in the evolution of telemedicine.

\section{Standards and Protocols}

It is widely presumed that telemedicine will adopt most of its standards and protocols as it becomes an integral part of medical practice and it will not be involved in their creation $^{8}$. However, telemedicine applications require high data rates and high bandwidth telecommunications that will drive the incorporation of appropriate standards and protocols into medical practice. In telemedicine, protocols and standards are needed to provide image intensive medical data from its creation, transmission to storage. These standard and protocols will provide timely access to specialty image data (e.g., X-rays, ultrasonic etc.) to the health care providers whatever their location. Existing medical standards setting bodies have defined a comprehensive set of protocols and standards for medical data managements and data transfer. These standards and protocols should provide the guideline for the selection and operation of data management networks for most telemedicine applications. The communication satellite network providers have to provide the quality of service required for the emerging telemedicine applications.

\section{Security}

Maintaining the confidentiality of medical data is a very serious issue in United States and several other countries. Secure communications are needed for telemedicine. In many countries it is less of an issue since access to quality medical care is a higher priority rather than legal issues.

\section{Others}

Medical malpractice and liability are also issues. The judgment of health care providers may be incorrect or brought into question based on poor transmission quality of typically very high resolution medical images or erroneous data transmission. Satellite transmission must provide the required standard of medical images and data accuracy for telemedicine to be feasible.

Training of individuals to operate telemedicine systems properly and efficiently is another concern.

There are concerns whether consumers will choose to utilize telemedicine services. Patient acceptance is high for telemedicine when compared with unavailability of medical care. However, recent surveys show that overall acceptance of telemedicine is low.

\section{Conclusions}

In the last two decades, numerous telemedicine demonstrations by communication satellites have clearly shown that medical information can be exchanged at a distance at National and Global level. During the demonstrations, users have benefited, however cost and accessability have not allowed them to use the technology permanently. Now, as the communication satellites define their role in the emerging NI//GII as evident by the several proposed and planned broadband satellite networks, it is likely that health care applications will grow. Several critical issues outlined in this paper must be resolved to achieve wide range acceptance of satellites in the health information infrastructure. 


\section{References}

1] The National Information Infrastructure: Agenda for Action, Information Infrastructure Task Force, September 1993 and The Global Information Infrastructure: Agenda for Cooperation, 1995.

2] Ausseresses, Antoine D., "Telecommunications Requirements for Telemedicine", Journal of Medical Systems, Vol. 19, No. 2, pp. 143-151, April 1995.

3] Bashshur, Rashid L., "Telemedicine Effects: Cost, Quality, and Access", Joumal of Medical Systems, Vol. 19, No. 2, pp. 81-91, April 1995.

4] Bashshur, Rashid L. and Jim Grigsby, "Position Paper: Telemedicine Effects: Cost, Quality, and Access", Journal of Medical Systems, Vol. 19, No. 2, pp. 79-80, April 1995.

5] Clemmer, Terry P., "The Role of Medical Informatics in Telemedicine", Journal of Medical Systems, Vol. 19, No. 1, pp. 47-58, February 1995.

6] Ferguson, Earl W., Charles R. Doarn, and John C. Scott, "Survey of Global Telemedicine", Journal Of Medical Systems, Vol. 19, No. 1, pp. 35-46, February 1995.

7] Garshnek, V., "Applications Of Space Communications Technology To Critical Human Needs: Rescue, Disaster Relief, And Remote Medical Assistancen, pp. 311-317.

8] Houtchens, Bruce A., Ace Allen, Terry P. Clemmer, Donald A. Lindberg, and Steinar Pedersen, "Telemedicine Protocols and Standards: Development and Implementation," Joumal of Medical Systems, Vol. 19, No. 2, pp. 93-119, April 1995.

9] Jarris, R. F., "Satellite Video System Aids Offshore Treatment", Health Management Technology, Vol. ??, No. ??, pp. 32-34, 1994.

10] Mandil, Salah H., "Telematics in Health Care in Developing Countries", Journal of Medical Systems, Vol. 19, No. 2, pp. 195-203, April 1995.

11] Mun, Seong K., Elsayed, Al M., Tohme, Walid G., and Wu, Y. Chris, "Teleradiology/Telepathology Requirements and Implementation", Joumal of Medical Systems, Vol. 19, No. 2, pp. 153-164, April 1995

12] Murakami, H., K. Shimizu, K. Tamamoto, T. Mikami, N. Hoshimiya, and K. Kondo, "Telemedicine Using Mobile
Satellite Communication", IEEE Transactions On Biomedical Engineering, Vol. 41, No. 5, pp. 488-497, 1994.

13] Orozco-Barbosa, L., A. Karmouch, N. D. Georganas, and M. Goldberg, "A Multimedia Interhospital Communications System For Medical Consultations", IEEE Journal On Selected Areas In Communications, Vol. 10, No. 7, pp. 1145-1157, 1992.

14] Padeken, D., D. Sotiriou, K. Boddy, and R. Gerzer, "Health Care in Remote Areas", Journal of Medical Systems, Vol. 19, No. 1, pp. 69-76, February 1995.

15] Perednia, D. A. and A. Allen, MD, "Telemedicine Technology and Clinical Applications," JAMA, Vol. 273, No. 6, 1995 .

16] Puskin, Dena S., "Opportunities and Challenges to Telemedicine in Rural America", Journal of Medical Systems, Vol. 19, No. 1, pp. 59-67, February 1995.

17] Puskin, Dena S. and Jay H. Sanders, "Telemedicine Infrastructure Development", Journal of Medical Systems, Vol. 19, No. 2, pp. 125-9, April 1995.

18] Schnepf, J. A., D. H. C. Du, E. R. Ritenour, and A. J. Fahrmann, "Building Future Medical Education Environments Over ATM Networks", Communications Of The $A C M$, Vol. 38, No. 2, pp. 54-69, 1995.

19] Zuzek, J. E., M. A. Cauley, and J. E. Hollansworth, "The Telemedicine Spacebridge Project - A Joint U.S./Russian Venture In Long Distance Medicine Via Satellite", NASA Technical Memorandum 106523, pp. 110, February-March 1994.

\section{Appendix: Web Pages}

"Arent Fox Telemedicine Home Page", http://www.arentfox.com/telemedicine.html, 1995.

Edwards, Jes, "Concept Overview", http://www.matmo.army.mil/pages/library/ papers/concept/concept.html, 1994.

Edwards, Jes, "Test Bed Plan", http://www.matmo.army.mil/pages/library/papers/ testBedPlan/testBedPlan.html, 1994.

Pinck, Deborah, "AMT Home Page", http://haagar.jpl.nasa.gov/ pinckamt.html. 
Raliski, Ed, "Telemedicine Home Page," http://isnet.is.wfu.edu/tmed-def.html.

Raliski, Ed, "Telemedicine Home Page", http://isnet.is.wfuedu/tmed-reason.html.

"RNSA 1994 Demonstration: DICOM/ATM in Telemedicine",

http://wnw:xray.hmc.psu.edu/schedules/demo_intro.html
Rowberg, Alan, MD, "UW ACTS Home Page", http:/hww.rad.washington.edu/ACTS.html.

"Equipment Album", http://www.matmo.army.millpages/library/photos/ equipment.html.

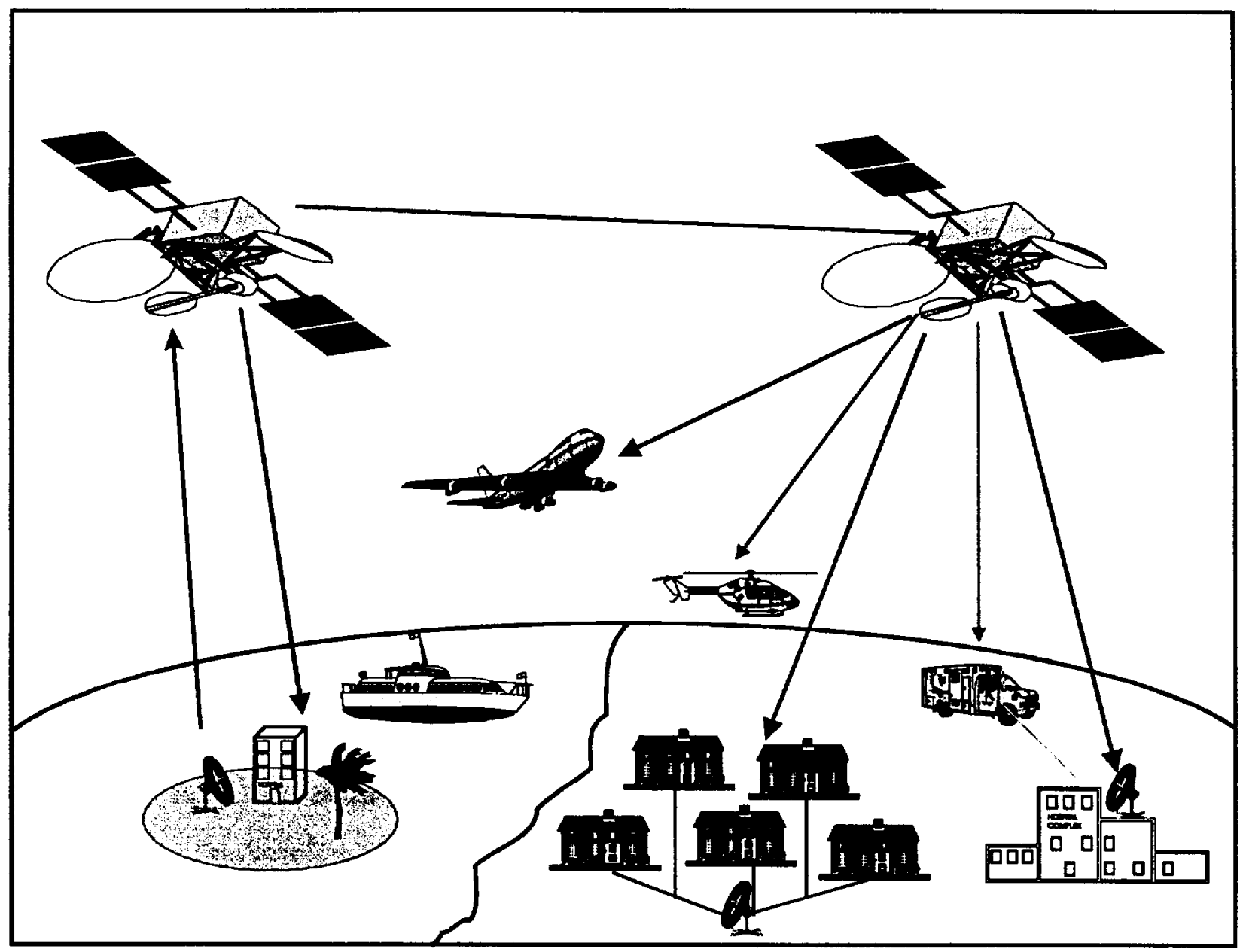

Figure 1 - Depiction of satellites operating in the Health Information Infrastructure 


\section{The Layered Approach}

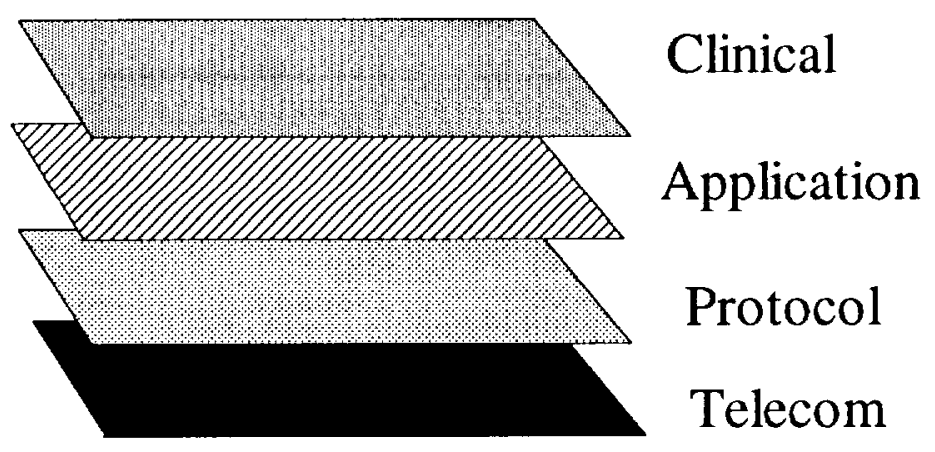

Figure 2 - An illustration of the various telecommunications layers with respect to telemedicine.

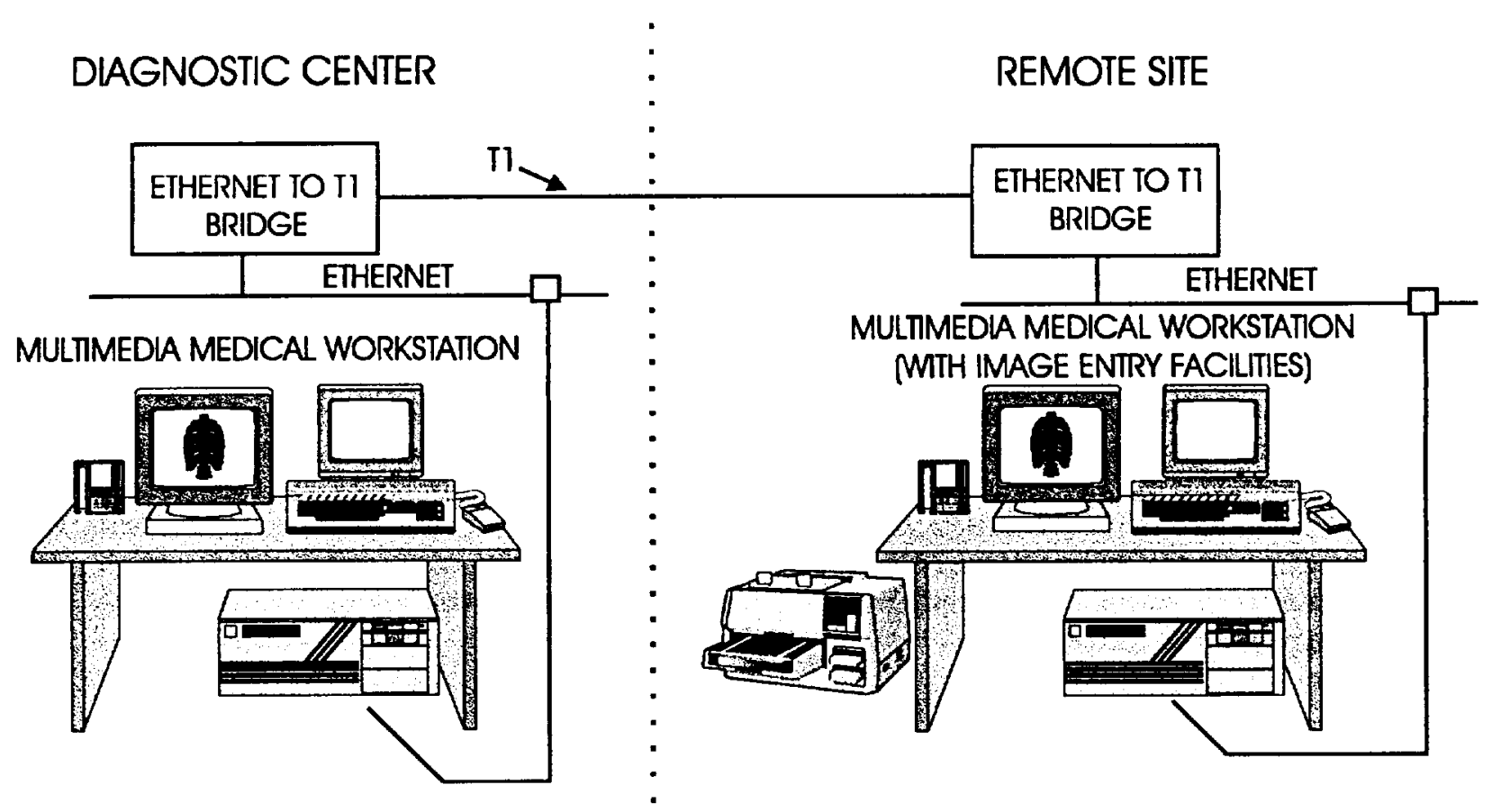

Figure 3- Two telemedicine multimedia workstations connected by a T1 link for telediagnostics 


\section{REPORT DOCUMENTATION PAGE}

Public reporting burden for this collactlon of hiormation ts estimated 10 average 1 houp per response, Including the time for revlewing instructons, caaching exiating data sources,


Davis Hlohway. Sulte 1204, Allington, VA 22202-4302, and to the Otilos of Management and Budget, Papenwork Reduction Project (0704-0188), Washington, DC 20503.

\begin{tabular}{|l|c|c|}
\hline 1. AGENCY USE ONLY (Leave blank) & $\begin{array}{c}\text { 2. REPOAT DATE } \\
\text { May } 1996\end{array}$ & $\begin{array}{r}\text { 3. REPORT TYPE AND DATES COVERED } \\
\text { Technical Memorandum }\end{array}$ \\
\hline
\end{tabular}

Communications Satellites in the National and Global Health Care

Information Infrastructure: Their Role, Impact, and Issues

6. AUTHOR(S)

WU-233-5A-5B

John E. Zuzek and Kul B. Bhasin

7. PERFORMING ORGANIZATION NAME(S) AND ADDRESS(ES)

8. PERFormina organization REPORT NUMBER

National Aeronautics and Space Administration

Lewis Research Center

Cleveland, Ohio 44135-3191

E-10245

9. SPONSORING/MONITORING AGENCY NAME(S) AND ADDRESS(ES)

10. SPONSORING/MONITORING AGENCY REPORT NUMBER

National Aeronautics and Space Administration

Washington, D.C. 20546-0001

NASA TM-107218

AIAA-96-0993

11. SUPPLEMENTARY NOTES

Prepared for the 16th International Communications Satellite Systems Conference cosponsored by AIAA, CASI, AAAF, DGLR, and IEICE, Washington, DC, February 25-29, 1996. Responsible person, John E. Zuzek, organization code 5610, (210 433-3469.

12.. DISTRIBUTIONAVAILABILTYY STATEMENT

12b. DISTRIBUTION CODE

Unclassified - Unlimited

Subject Categories 17 and 32

This publication is available from the NASA Center for AeroSpace Information, (301) 621-0390.

13. ABSTRACT (Moximum 200 mords)

Health care services delivered from a distance, known collectively as telemedicine, are being increasingly demonstrated on various transmission media. Telemedicine activities have included diagnosis by a doctor at a remote location, emergency and disaster medical assistance, medical education, and medical informatics. The ability of communications satellites to offer communication channels and bandwidth on demand, connectivity to mobile, remote and under served regions, and global access will afford them a critical role for telemedicine applications within the National and Global Information Infrastructure (NILGII). The importance that communications satellites will have in telemedicine applications within the NII/GII, the differences in requirements for NII vs. GII, the major issues such as interoperability, confidentiality, quality, availability, and costs, and preliminary conclusions for future usability based on the review of several recent trails at national and global levels are presented.

\begin{tabular}{|c|c|}
\hline 14. SUBJECT TERMS \\
Telemedicine; Satellite communications; Information infrast \\
$\begin{array}{c}\text { 17. SECURTY CLASSIFICATION } \\
\text { OF REPORT } \\
\text { Unclassified }\end{array}$ & $\begin{array}{c}\text { 18. SECURTY CLASSIFICATION } \\
\text { OF THIS PAGE } \\
\text { Unclassified }\end{array}$ \\
\hline
\end{tabular}

19. SECURTY CLASSIFICATION OF ABSTRACT Unclassified
5. NUMBER OF PAGES

11

16. PRICE CODE

A03
20. LIMTTATION OF ABSTRACT 
National Aeronautics and

Space Administration

Lewis Research Center

21000 Brookpark Rd.

Cleveland, $\mathrm{OH}$ 44135-3191

Official Business

Penalty for Private Use $\$ 300$

POSTMASTER: If Undeliverable - Do Not Return 